\title{
An Advanced Shell Theory Based Tire Model
}

REFERENCE: Bozdog, D. and Olson, W. W., "An Advanced Shell Theory Based Tire Model," submitted for presentation at the 2004 Tire Society meeting, and for consideration for publication in the journal Tire Science and Technology.

\begin{abstract}
The objective of this paper is to investigate a class of general tire models that provides results suitable for usage in vehicle dynamics. Tire models currently used for vehicle dynamic analyses are overly simplistic (springs, a spring and damper combination or semi-elastic substance) or based on curve fits of experimental data. In contrast, the tire models used by major tire companies are extremely complex with solutions possible only by finite element analysis. Between these two extremes exists the potential for an elasticity based shell theory tire model. Micro-mechanics and composite laminate theories provide an integrated approach to the macroscopic behavior of the tire carcass and the tread support plies. This methodology has the capability of including centrifugal and friction forces. Finite difference methods are applied that produce reliable and accurate solutions of the tire response.
\end{abstract}

\section{Table of Nomenclature}

$\mathrm{e}_{\phi}, \mathrm{e}_{\xi}, \mathrm{e}_{\mathrm{z}}-$ unit vectors

$\mathrm{h}_{\mathrm{k}}$ - layer thickness

$\mathrm{p}_{\phi}, \mathrm{p}_{\xi}, \mathrm{p}_{\mathrm{z}}-$ surface load components

$\mathrm{n}-$ number of layers

$\mathrm{u}, \mathrm{v}, \mathrm{w}-$ displacements components

A, B - Lamé parameters

$\mathrm{A}_{\mathrm{ij}}, \mathrm{B}_{\mathrm{ij}}, \mathrm{D}_{\mathrm{ij}}-$ stiffness coefficients

$\mathrm{K}_{\phi}, \mathrm{K}_{\xi}, \mathrm{K}_{\phi \xi}$ - change of curvature

$\mathrm{N}_{\phi}, \mathrm{N}_{\xi}, \mathrm{N}_{\phi \xi}-$ force resultants

$\mathrm{M}_{\phi}, \mathrm{M}_{\xi}, \mathrm{M}_{\phi \xi}$ - moment resultants

$\mathrm{M}^{(\phi)}, \mathrm{M}^{(\xi)}-$ moment resultants

$\mathrm{Q}_{\mathrm{ij}}$ - reduced stiffness matrix components

$\mathrm{Q}_{\phi}, \mathrm{Q}_{\xi}$ - shearing forces

$\mathrm{R}_{1}, \mathrm{R}_{2}$ - radii of curvature

$\mathrm{T}^{(\phi)}, \mathrm{T}^{(\xi)}$ - force resultants

$\varepsilon_{\phi}, \varepsilon_{\xi}, \gamma_{\phi \xi}$ - strain components

$\phi, \xi, \mathrm{z}$ - curvilinear coordinate system

$\sigma_{\phi}, \sigma_{\xi}, \tau_{\phi \xi}-$ stress components 


\section{Introduction}

The single most important external excitation of a vehicle is the profile of the terrain. The tire and suspension system translates the profile into motions of the vehicle mass. Lumped parameter springs and dampers model the suspension system relatively well. However, such systems fail to capture the observed tire performance in vehicle dynamics models. In very simple models, the tire is modeled as a stiff spring of a somewhat arbitrary spring rate. Representations that are more complex admit different configurations of springs and dampers representing the Maxwell and Voight tire viscoelastic responses. Even more complex formulations are represented by multiple spring models including the "brush" model. However, experimental results have not in general confirmed the accuracy of these models. Another approach is to measure and curve fit experimental data. This method, particularly with the four-parameter Pacejka model, is quite successful in producing reliable results. However, the results are very specific to the tire measured and are difficult to generalize.

There is a need to have a tire model based on the principles of physics of the tire composite model that correctly translates the profile and properties of a terrain surface into forces experienced at the axle of a vehicle as the vehicle moves on terrain. The development of scientific tire models is a requirement for significant improvements of modeling quality. The ability the model to be predictive over a broad class of movements and profiles is essential to modeling future vehicles and designing future vehicles using the advanced capabilities of the computer.

In this vein, this paper describes the work performed to develop a general shell theory model of a tire. The initial work builds on a small deformation linear shell theory first successfully applied by Brewer in the early 1970's. However Brewer was unable to progress on his model as the computational capabilities that existed at that time were limited as compared to computers today. This model was extended to include twisting that could not be modeled in the 1970's. Furthermore, the work described in this paper builds towards a large deformation, nonlinear tire model, which is under investigation.

\section{Tire Model Formulation}

A curvilinear coordinate system was constructed on the tire mid-surface. This is represented in Fig. 1. The local coordinates used to describe the tire system are $\phi$, the meridian direction that varies along the cross section of the tire, $\xi$, the circumferential direction, and $z$, normal to the surface. Note that the apex of $\phi$ is at the tire rim. This avoids a mathematical discontinuity if the apex were in the center of the cross section as it is in most toroid shell analyses. The global system of coordinates is represented by $\mathrm{XYZ}$. 

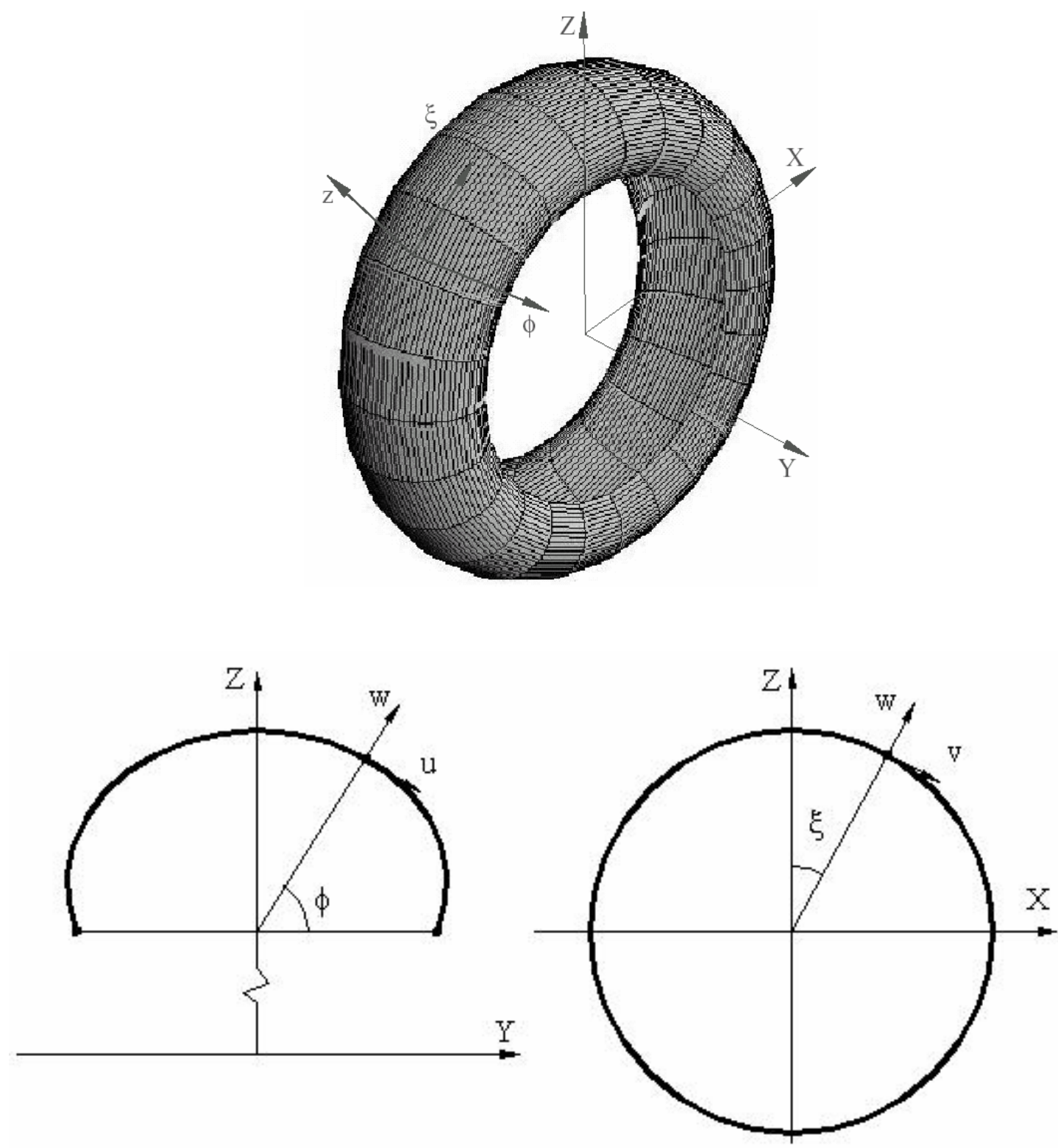

Figure 1: Tire coordinate system and displacements

The geometric strain-displacement equations from shell theory are: 


$$
\begin{aligned}
\varepsilon_{\phi} & =\frac{1}{A}\left(\frac{\partial u}{\partial \phi}\right)+\frac{1}{A B}\left(\frac{\partial A}{\partial \xi}\right) v+\frac{w}{R_{1}} \\
\varepsilon_{\xi} & =\frac{1}{B}\left(\frac{\partial v}{\partial \xi}\right)+\frac{1}{A B}\left(\frac{\partial B}{\partial \phi}\right) u+\frac{w}{R_{2}} \\
\gamma_{\phi \xi} & =\frac{B}{A} \frac{\partial}{\partial \phi}\left(\frac{v}{B}\right)+\frac{A}{B} \frac{\partial}{\partial \xi}\left(\frac{u}{A}\right) \\
K_{\phi} & =\frac{1}{A} \frac{\partial}{\partial \phi}\left(\frac{u}{R_{1}}+\frac{1}{A} \frac{\partial w}{\partial \phi}\right)+\frac{1}{A B} \frac{\partial A}{\partial \xi}\left(\frac{v}{R_{2}}+\frac{1}{B} \frac{\partial w}{\partial \xi}\right) \\
K_{\xi} & =\frac{1}{B} \frac{\partial}{\partial \xi}\left(\frac{v}{R_{2}}+\frac{1}{B} \frac{\partial w}{\partial \xi}\right)+\frac{1}{A B} \frac{\partial B}{\partial \phi}\left(\frac{u}{R_{1}}+\frac{1}{A} \frac{\partial w}{\partial \phi}\right) \\
K_{\phi \xi} & =\frac{1}{A B}\left(-\frac{1}{A} \frac{\partial A}{\partial \xi} \frac{\partial w}{\partial \phi}-\frac{1}{B} \frac{\partial B}{\partial \phi} \frac{\partial w}{\partial \xi}+\frac{\partial^{2} w}{\partial \phi \partial \xi}\right)-\frac{A}{B R_{1}} \frac{\partial}{\partial \xi}\left(\frac{u}{A}\right)-\frac{B}{A R_{2}}\left(\frac{v}{B}\right)
\end{aligned}
$$

where $\mathrm{A}$ and $\mathrm{B}$ are the Lamé parameters, $\mathrm{R}_{1}$ and $\mathrm{R}_{2}$ are the principal radii, $u, v$, and $w$ are displacement components and $\varepsilon_{\phi}, \varepsilon_{\xi}$, and $\gamma_{\phi \xi}$, are strain components and $K_{\phi}, K_{\xi}$, and $K_{\phi \xi}$ are changes in shell curvature.

The Lamé parameters and radii of curvature are related to one another by Codazzi and Gauss conditions:

$$
\frac{\partial}{\partial \xi}\left(\frac{A}{R_{1}}\right)=\frac{1}{R_{2}} \frac{\partial A}{\partial \xi} \quad \frac{\partial}{\partial \phi}\left(\frac{B}{R_{2}}\right)=\frac{1}{R_{1}} \frac{\partial B}{\partial \phi}
$$

and

$$
\frac{\partial}{\partial \phi}\left(\frac{1}{A} \frac{\partial B}{\partial \phi}\right)+\frac{\partial}{\partial \xi}\left(\frac{1}{B} \frac{\partial A}{\partial \xi}\right)=-\frac{A B}{R_{1} R_{2}}
$$

The three dimensional carcass of the tire is reduced to two dimensions by modeling all loadings at the middle surface. A major assumption in developing a shell theory is that the critical dimensions of length and radii are much greater than the thickness of the shell. This is true with a tire. The carcass thickness for an automotive tire is of the order of a few tenths of an inch while the radii involved are greater than 4 inches. The assumption is less valid for the tread, which is of the order of an inch but still within the range of acceptable error. Another measure is that the thickness be small compared to the lengths. The meridian lengths for an automotive tire are greater than 8 inches while the circumferential lengths are much larger. Consequently, forces and moments load the middle-surface and are placed in at the middle surface. The stress resultants and stress couples are presented in Fig. 2. 


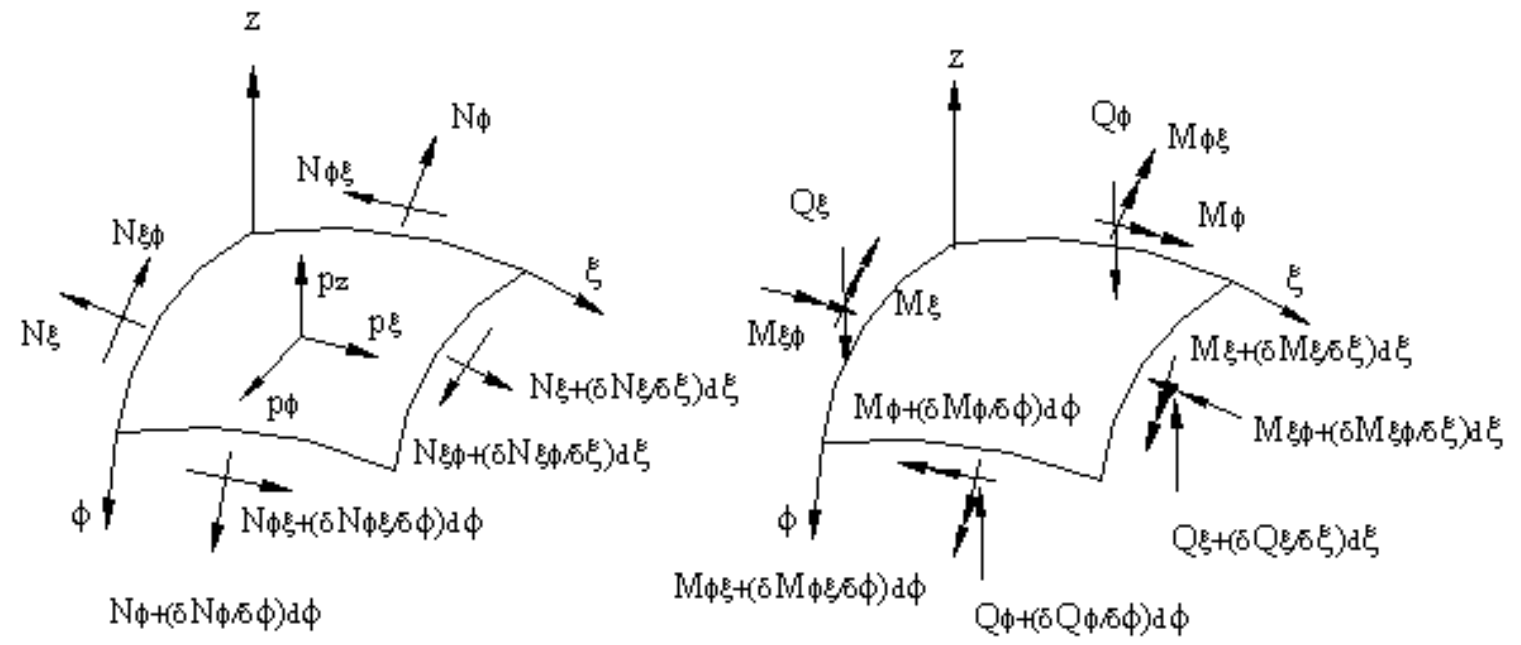

Figure 2: Shell stress resultants and stress couples with transverse forces resultant

The internal forces and moments acting on the differential element will be replaced by equivalent couple force systems consisting of resultant forces $\mathrm{T}^{(\phi)}$ and $\mathrm{T}^{(\xi)}$ and resultant couples $\mathrm{M}^{(\phi)}$ and $\mathrm{M}^{(\xi)}$ as shown in Fig. 3.



Figure 3: Couple-Force System

The forces and moments in the in the $\phi$ direction are

$$
\begin{aligned}
-T^{(\phi)} & =-\left(N_{\phi \xi} e_{\xi}+N_{\phi} e_{\phi}+Q_{\phi} e_{z}\right) B d \xi \\
-M^{(\phi)} & =-\left(M_{\phi \xi} e_{\phi}-M_{\phi} e_{\xi}\right) B d \xi
\end{aligned}
$$

The forces and moments in the in the $\xi$ direction are

$$
\begin{aligned}
-T^{(\xi)} & =-\left(N_{\xi} e_{\xi}+N_{\xi \phi} e_{\phi}+Q_{\xi} e_{z}\right) A d \phi \\
-M^{(\xi)} & =-\left(M_{\xi} e_{\phi}-M_{\xi \phi} e_{\xi}\right) A d \phi
\end{aligned}
$$


The resultant forces and moments acting on the opposite faces $\phi$ and $\phi+d \phi$, as well as $\xi$ and $\xi+d \xi$ have opposite signs. The surface load is expressed as a distributed pressure:

$$
p=p_{\phi} e_{\phi}+p_{\xi} e_{\xi}+p_{z} e_{z}
$$

When placed in equilibrium, the system of equations produced is

$$
\begin{aligned}
\frac{\partial}{\partial \phi}\left(N_{\phi} B\right)+\frac{\partial}{\partial \xi}\left(N_{\xi \phi} A\right)+N_{\phi \xi} \frac{\partial A}{\partial \xi}-N_{\xi} \frac{\partial B}{\partial \phi}+Q \frac{A B}{R_{1}}+p_{\phi} A B & =0 \\
\frac{\partial}{\partial \xi}\left(N_{\xi} A\right)+\frac{\partial}{\partial \phi}\left(N_{\phi \xi} B\right)+N_{\xi \phi} \frac{\partial B}{\partial \phi}-N_{\phi} \frac{\partial A}{\partial \xi}+Q_{\xi} \frac{A B}{R_{2}}+p_{\xi} A B & =0 \\
\frac{\partial}{\partial \phi}\left(Q_{\phi} B\right)+\frac{\partial}{\partial \xi}\left(Q_{\xi} A\right)-N_{\phi} \frac{A B}{R_{1}}-N_{\xi} \frac{A B}{R_{2}}+p_{z} A B & =0 \\
\frac{\partial}{\partial \phi}\left(M_{\phi \xi} B\right)+\frac{\partial}{\partial \xi}\left(M_{\xi} A\right)-M_{\phi} \frac{\partial A}{\partial \xi}+M_{\xi \phi} \frac{\partial B}{\partial \phi}-Q_{\xi} A B & =0 \\
\frac{\partial}{\partial \xi}\left(M_{\xi \phi} A\right)+\frac{\partial}{\partial \phi}\left(M_{\phi} B\right)-M_{\xi} \frac{\partial B}{\partial \phi}+M_{\phi \xi} \frac{\partial A}{\partial \xi}-Q_{\phi} A B & =0 \\
N_{\phi \xi}-N_{\xi \phi}+\frac{M_{\phi \xi}}{R_{1}}-\frac{M_{\xi \phi}}{R_{2}} & =0
\end{aligned}
$$

A typical tire structure consists of a system of layers bonded together. The individual plies have various angles in a prescribed sequence. The classical lamination theory assumes that the orthotropic layers are perfectly bonded together with an infinitely thin bond line and the deformations across the bond line are continuous [1-3]. Generally, the multi-ply systems twist and bend when subjected to simple tensile load. An out of plane twisting of the laminate structure is observed when two plies with opposite angle are bonded together. The result is a combination of bending and stretching of the laminate.

The development of the constitutive properties for the tire follows Walters [4]. The constitutive equations for an anisotropic layer have the following expression:

$$
\left[\begin{array}{c}
\sigma_{\phi} \\
\sigma_{\xi} \\
\tau_{\phi \xi}
\end{array}\right]_{k}=\left[\begin{array}{lll}
\bar{Q}_{11} & \bar{Q}_{12} & \bar{Q}_{16} \\
\bar{Q}_{12} & \bar{Q}_{22} & \bar{Q}_{26} \\
\bar{Q}_{16} & \bar{Q}_{26} & \bar{Q}_{66}
\end{array}\right]\left[\begin{array}{c}
\varepsilon_{\phi}^{0} \\
\varepsilon_{\xi}^{0} \\
\gamma_{\phi \xi}^{0}
\end{array}\right]_{k}+z\left[\begin{array}{lll}
\bar{Q}_{11} & \bar{Q}_{12} & \bar{Q}_{16} \\
\bar{Q}_{12} & \bar{Q}_{22} & \bar{Q}_{26} \\
\bar{Q}_{16} & \bar{Q}_{26} & \bar{Q}_{66}
\end{array}\right]\left[\begin{array}{c}
K_{\phi} \\
K_{\xi} \\
K_{\phi \xi}
\end{array}\right]_{k}
$$

The stress resultants and moment resultants per unit width of the cross-section acting at a point in the laminate are determined by integration of the stresses for each ply. Separating the continuous integrals over each of the $n$ layers, the stress resultants are expressed as the sum of $n$ simple integrals. 


$$
\left[\begin{array}{c}
N_{\phi} \\
N_{\xi} \\
N_{\phi \xi}
\end{array}\right]=\sum_{k=1}^{n} \int_{h_{k-1}}^{h_{k}}\left[\begin{array}{c}
\sigma_{\phi} \\
\sigma_{\xi} \\
\tau_{\phi \xi}
\end{array}\right] d z \quad\left[\begin{array}{c}
M_{\phi} \\
M_{\xi} \\
M_{\phi \xi}^{\xi}
\end{array}\right]=\sum_{k=1}^{n} \int_{h_{k-1}}^{h_{k}}\left[\begin{array}{c}
\sigma_{\phi} \\
\sigma_{\xi} \\
\tau_{\phi \xi}
\end{array}\right] z d z
$$

Finally, the constitutive equations are:

$$
\left[\begin{array}{c}
N_{\phi} \\
N_{\xi} \\
N_{\phi \xi} \\
M_{\phi} \\
M_{\xi} \\
M_{\phi \xi}
\end{array}\right]=\left[\begin{array}{llllll}
A_{11} & A_{12} & A_{16} & B_{11} & B_{12} & B_{16} \\
A_{12} & A_{22} & A_{26} & B_{12} & B_{22} & B_{26} \\
A_{16} & A_{26} & A_{66} & B_{16} & B_{26} & B_{66} \\
B_{11} & B_{12} & B_{16} & D_{11} & D_{12} & D_{16} \\
B_{12} & B_{22} & B_{26} & D_{12} & D_{22} & D_{26} \\
B_{16} & B_{26} & B_{66} & D_{16} & D_{26} & D_{66}
\end{array}\right]\left[\begin{array}{c}
\varepsilon_{\phi} \\
\varepsilon_{\xi} \\
\gamma_{\phi \xi} \\
K_{\phi} \\
K_{\xi} \\
K_{\phi \xi}
\end{array}\right]
$$

with:

$$
\begin{aligned}
A_{i j} & =\sum_{k=1}^{n}\left[\bar{Q}_{i j}\right]_{k}\left(h_{k}-h_{k-1}\right) \\
B_{i j} & =\frac{1}{2} \sum_{k=1}^{n}\left[\bar{Q}_{i j}\right]_{k}\left(h_{k}^{2}-h_{k-1}^{2}\right) \\
D_{i j} & =\frac{1}{3} \sum_{k=1}^{n}\left[\bar{Q}_{i j}\right]_{k}\left(h_{k}^{3}-h_{k-1}^{3}\right)
\end{aligned}
$$

where:

$\left[A_{i j}\right]$ is associated with in-plane extension, contraction and shear, $\left[B_{i j}\right]$ is associated coupling effects between bending and stretching, $\left[D_{i j}\right]$ is associated with bending and twisting.

The system of geometric strain-displacement equations, equilibrium equations, constitutive equations, and boundary conditions describes completely the behavior of the tire. Two additional simplifications, $N_{\phi \xi}=N_{\xi \phi}$ and $M_{\phi \xi}=M_{\xi \phi}$, reduces the system to 17 equations and 17 unknowns:

$$
\varepsilon_{\phi}, \varepsilon_{\xi}, \gamma_{\phi \xi}, K_{\phi}, K_{\xi}, K_{\phi \xi}, N_{\phi}, N_{\xi}, N_{\phi \xi}, M_{\phi}, M_{\xi}, M_{\phi \xi}, Q_{\phi}, Q_{\xi}, u, v, w .
$$

Strategically, this system can be solved in two ways. In the first method, the forces and moments in the equilibrium equations are replaced by the displacement components, which (after applying the constitutive relationships) results in an eight order, three partial differential equations, system that is solved for the displacements. In the second method, the displacements are replaced in the geometric compatibility equations by the forces and 
moments resulting in an eight order, six partial differential equations, system, which is simultaneously solved for the forces, and moments. In this work, the first method is employed.

\section{Numerical method}

It is highly unlikely that an analytical solution to the equations exist due to the nonlinear terms. Therefore numerical methods must be used to solve the equations. In this analysis a finite difference approach was selected as the geometry is relatively simple. The partial differentials were replaced with their central difference approximations:

$$
\begin{aligned}
\frac{\partial f}{\partial \phi} & =\frac{f(i+1, j)-f(i-1, j)}{2 \Delta \phi} \\
\frac{\partial f}{\partial \xi} & =\frac{f(i, j+1)-f(i, j-1)}{2 \Delta \phi} \\
\frac{\partial^{2} f}{\partial \phi^{2}} & =\frac{f(i+1, j)-2 f(i, j)+f(i,-1 j)}{2 \Delta \xi} \\
\frac{\partial^{2} f}{\partial \xi^{2}} & =\frac{f(i, j+1)-2 f(i, j)+f(i, j-1)}{2 \Delta \xi} \\
\frac{\partial^{2} f}{\partial \phi \partial \xi} & =\frac{f(i+1, j+1)-f(i-1, j+1)-f(i+1, j-1)+f(i-1, j-1)}{4 \Delta \phi \Delta \xi}
\end{aligned}
$$

The program code was written using Mathematica ${ }^{\mathrm{TM}}$ software. The advanced performance of the symbolic computation of the software package and the advanced mathematical tools offered were the main reasons of choosing this software package. An additional benefit Mathematica ${ }^{\mathrm{TM}}$ is the ease of converting the finished program into $\mathrm{C}$ for faster performance.

\section{Results}

The Brewer tire cross section with a stiffness matrix corresponding to a radial tire was analyzed using various internal pressure configurations.[5] Brewer was chosen as it established an effective standard by which to compare this work. The Brewer tire is a 32 X 8.8 Type VII aircraft tire inflated to 95 psi. The stiffness coefficients are

$$
\left[\begin{array}{llllll}
A_{11} & A_{12} & A_{16} & B_{11} & B_{12} & B_{16} \\
A_{12} & A_{22} & A_{26} & B_{12} & B_{22} & B_{26} \\
A_{16} & A_{26} & A_{66} & B_{16} & B_{26} & B_{66} \\
B_{11} & B_{12} & B_{16} & D_{11} & D_{12} & D_{16} \\
B_{12} & B_{22} & B_{26} & D_{12} & D_{22} & D_{26} \\
B_{16} & B_{26} & B_{66} & D_{16} & D_{26} & D_{66}
\end{array}\right]=\left[\begin{array}{cccccc}
20.04 & 40.36 & 0 & 0 & 0 & 0.525 \\
40.36 & 93.51 & 0 & 0 & 0 & 1.249 \\
0 & 0 & 38.54 & 0.525 & 1.249 & 0 \\
0 & 0 & 0.525 & 0.111 & 0.224 & 0 \\
0 & 0 & 1.249 & 0.224 & 0.519 & 0 \\
0.525 & 1.249 & 0 & 0 & 0 & 0.214
\end{array}\right] \cdot 10^{2}
$$


where $A_{i j}\left[\mathrm{lb} / \mathrm{in}^{2}\right], B_{i j}[\mathrm{lb}]$, and $D_{i j}[\mathrm{in} * \mathrm{lb}]$.

The boundary conditions were set along the rim edges. Displacements were fixed at zero as the tire bead is assumed rigid under the deformation levels imposed. The tire wall was allowed to rotate with the bead as a center. Rotation normal to the bead was also fixed at zero.

Displacements of the cross-section at 95 psi for both this model and Brewer's model are shown Figure 4. At the crown of the tire, the results are exact. However, in the sidewall, there is a pronounced difference between these results and Brewer's results. We believe the results of this model are more accurate as Brewer's model does not significantly deviate from the original shape until midway along the carcass.

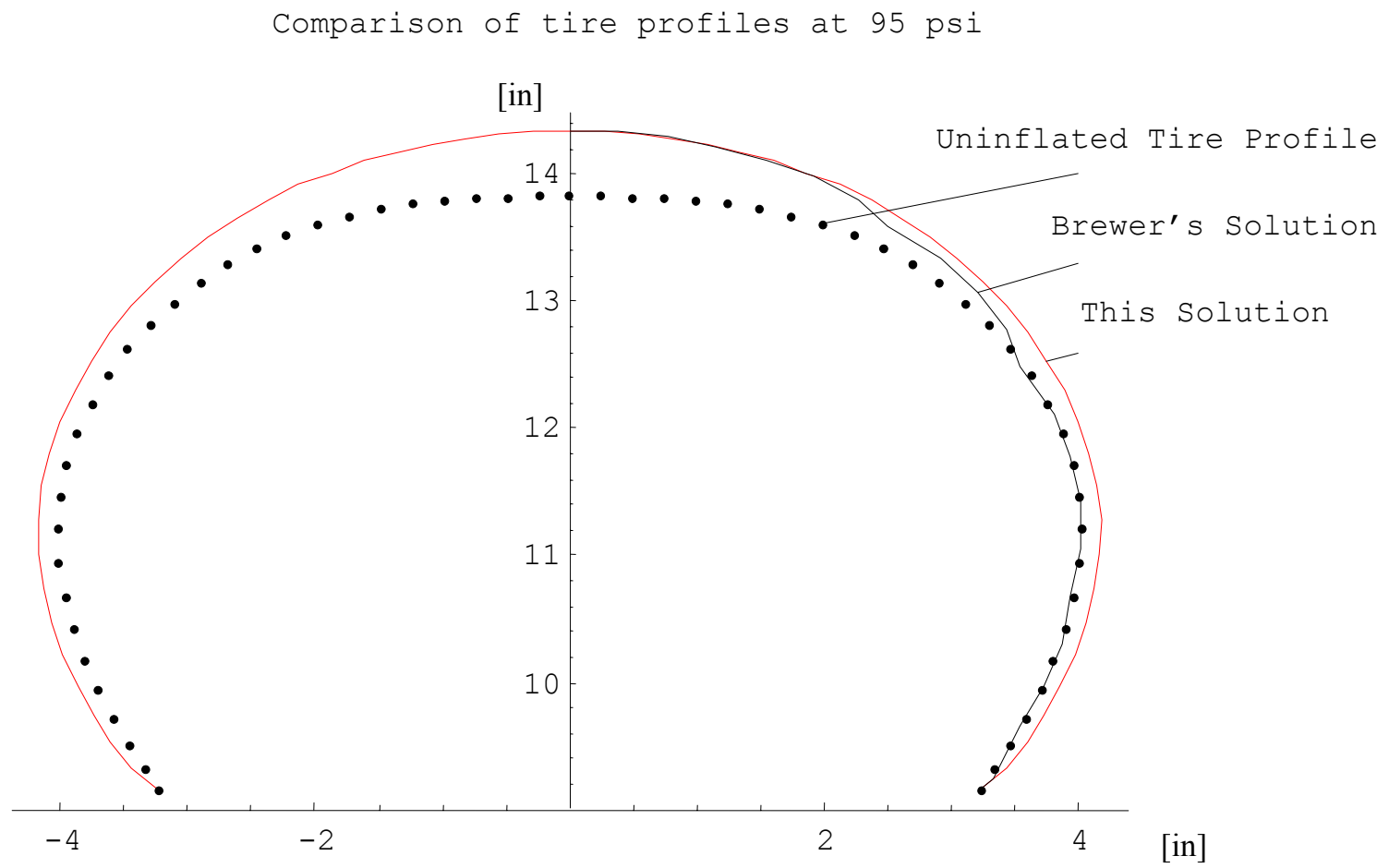

Figure 4: Cross sections comparing solutions

$\phi$ along a cross section was set to 61 nodes ( 4-degree increments). While Brewer did not perform computations in the $\xi$ direction, the value of $\Delta \xi$ was set at 2-degree increments. The computational time needed to produce these results using a Windows based machine with a Pentium 4, 3.4 GHz processor, was 121 seconds for a solution.

The analysis of deformations is presented in Figure 5. The centerline of the cross section is at node 30. This corresponds to an angle of 90 degrees about the $\mathrm{Y}$ axis of the problem. Both $u$ and $v$ change signs because the direction changes from positive to negative as the analysis traverses the midpoint of the cross section. Also note that the deformations are 
small as expected with in the tire section. The outward deformation represented by the $w$ direction is greater, also as expected. The maximum value is achieved along the $\mathrm{Z}$ axis as the tire boundary condition at the rim is fixed. Brewer did not present the deformations in his work.

Figure 6 shows the representative strain and shear components. The strain in the meridian, $\varepsilon_{\phi}$, is greatest in the sidewall of the tire structure while the hoop strain, $\varepsilon_{\xi}$, is greatest at the crown of the tire. The shear in the surface, $\gamma_{\phi \xi}$, is quite small and decreases from the tire bead. The strain values are very similar to Brewer in shape although about an order of magnitude less.

Figure 7 presents the membrane forces and moments computed. The forces computed here differ significantly from those reported by Brewer. It is quite possible that there is typographical error in the labeling of his graph, as the forces listed in Appendix of his earlier published thesis [8] are two magnitudes greater than those presented graphically in his paper [5]. The membrane forces appear to be correct in both shape and magnitude; however, the moments in the sidewalls are somewhat unstable in these computations. The cause for the instability has not been identified although they do not have a major effect on the solution found. It should be noted that the magnitude fluctuations appear in Brewer's work as well although not as pronounced as here. The inter-ply forces grow to a maximum in the tread region while the twist reaches a minimum value in the tire crown.

Deformations at 95 psi
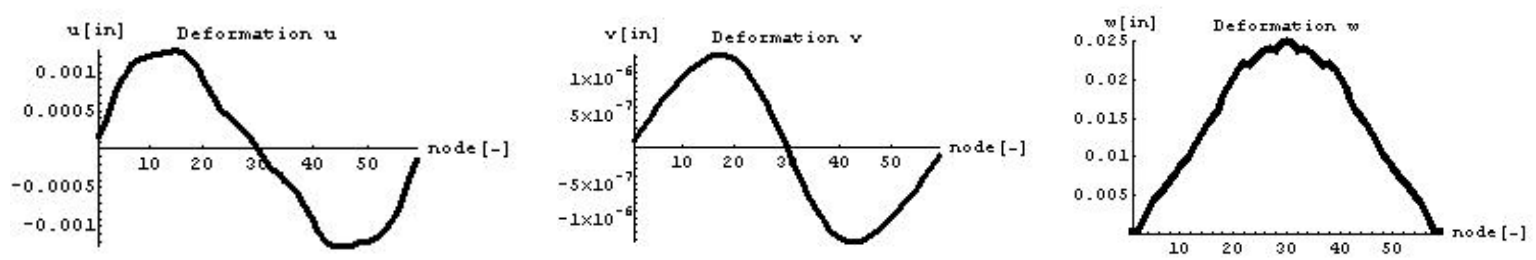

Figure 5: Analysis of deformations
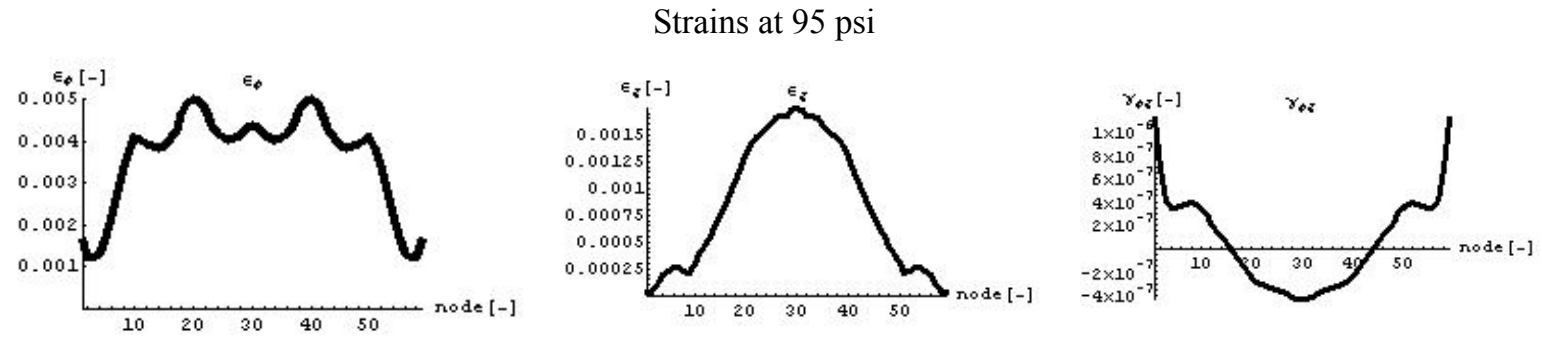

Figure 6: Analysis of strains and shear

Forces and Moments at 95 psi 



Figure 7: Analysis of forces and moments

\section{Conclusions}

The results paralleled Brewer's work, although the solution method used were considerably different. Where as Brewer solved the equations with Kalnin's shooting methods in a two dimensional framework, this analysis used a direct finite difference method. We believe the model as presented is reasonably correct in predicting the small deformations of a tire as it has been validated against a well known and public accessible benchmark in tire science.

The success in solving a linear shell theory in three dimensions using a finite difference scheme is a major step towards completing the overall objectives of this project. The method shown here is suitable for extending the work to large strain, nonlinear shell model whereas a solution method such as Kalnin's would have proved difficult in multiple dimensions. This project, while still in progress, demonstrates that physics based tire model can be solved within reasonable computation time and power to provide force inputs to a vehicle dynamics model. Future work planned at this time includes building and solving the finite strain model and imposing the road forces including friction and load onto the tire.

\section{References}

[1] Vasiliev, V. V., Morozov, E. V., Mechanics and Analysis of Composite Materials, Elsevier, Netherlands, 2001.

[2] Gürdal, Z., Haftka, R. T., Hajela, P., Design and Optimization of Laminated Composite Materials, John Wiley \& Sons, New York, 1999.

[3] Mallick, P. K., Fiber-Reinforced Composites: Materials, Manufacturing, and Design, Marcel Dekker, New York, 1988. 
[4] Walter, J. D., "Cord Reinforced Rubber," Chapter 3 of Mechanics of Pneumatic Tires, U.S. Department of Transportation, National Highway Traffic Safety Administration, D.C., 1981.

[5] Brewer, H. K., "Tire Stress and Deformation form Composite Theory," Tire Science and Technology, Vol. 1, No. 1, Feb 1973, pp 47-75.

[6] Ventzel, E., Krauthammer, T., Thin Plates and Shells: Theory, Analysis, and Applications, Marcel Dekker, New York, 2001.

[7] Wolfram, S., The Mathematica Book, $3^{\text {rd }}$ ed., Wolfram Media, Cambridge University Press, 1996.

[8] Brewer, H. K., Stresses and Deformations in Multi-Ply Aircraft Tires Subject to Inflation Pressure Loading, Air Force Flight Dynamics Laboratory, WrightPatterson Air Force Base, Ohio, 1970. 